\title{
Prediction of Bus-Transfer Switching in Future HVDC Substations
}

\section{Journal Article}

Author(s):

Ritter, Andreas (D); Franck, Christian (D)

Publication date:

2018-06

Permanent link:

https://doi.org/10.3929/ethz-b-000205822

Rights / license:

In Copyright - Non-Commercial Use Permitted

Originally published in:

IEEE Transactions on Power Delivery 33(3), https://doi.org/10.1109/tpwrd.2017.2764386 


\title{
Prediction of Bus-Transfer Switching in Future HVDC Substations
}

\author{
Andreas Ritter, Student Member, IEEE, and Christian M. Franck, Senior Member, IEEE
}

\begin{abstract}
Prior research has characterized bus-transfer switching capabilities of disconnectors commonly found in ac gas insulated switchgear (GIS) by means of a limit performance prediction. A large number of dc bus-transfer tests was conducted on a modified bus-transfer test setup in order to study the applicability of these findings for the use of disconnectors in future HVdc substations. It was found that the ac extinction criterion based solely on the instantaneous recovery voltage and the expected arc voltage at current zero is also valid for dc bustransfer switching. However, due to the high frequency harmonics inherent to HVdc power transmission, the process of reaching the initial current zero and the extinction of the switching arc at current zero have been discovered to be independent to a certain degree. For negligible harmonic content of the switching current, arcing times below $20 \mathrm{~ms}$ have been predicted for reasonable sizes of substations and realistic dc current magnitudes, which is certainly within the capabilities of existing ac disconnectors. The previously devised limit performance prediction can be applied to gauge influences of different harmonic current contents and small modifications to the disconnector in simulation by applying the reignition criterion at the initial and all subsequent current zeros. Ultimately, however, exact knowledge of frequency and magnitude of the harmonic currents is needed in order to perform accurate predictions.
\end{abstract}

Index Terms-HVdc, disconnector, bus-transfer, currentinterruption, gas insulated substations, testing, iec

\section{INTRODUCTION}

$\mathbf{I}$ NCREASING demand for integrating renewable energy sources into existing ac power grids has led to a number of propositions for $\mathrm{HVdc}$ multi-terminal grids in recent years. With the advances in power electronics enabling voltage source converters (VSC) at high dc voltages, multiterminal HVdc grids have become a reality when the Nan'ao HVdc system was commissioned by China Southern Grid in December 2013 [1], [2]. Identified as one of the key enabling technologies for large multi-terminal grids, $\mathrm{HVdc}$ circuit breakers (CB) have seen tremendous research effort in the past decade (cf. [3]). The installation of the first production models is currently underway in the Zhoushan five terminal grid ([4], [5]) after manufacturers started presenting functioning prototypes ([6], [7]) a few years ago. Due to the substantial costs of first-generation $\mathrm{HVdc} \mathrm{CB}$, the number of $\mathrm{CB}$ planned to be employed in any multi-terminal grid is designed to be minimal, i.e. one $\mathrm{CB}$ per substation feeder or fewer.

In terms of insulation medium employed in an HVdc substation, gas insulated switchgear (GIS) offers advantages over

A. Ritter and C. M. Franck are with the Power Systems and High Laboratories (EEH), ETH Zurich, Zurich 8092, Switzerland (e-mail: rittera@ethz.ch).

This work was financially supported by ABB Switzerland Ltd.

Manuscript received ?; revised? air insulated switchgear (AIS) such as reduced size and immunity against pollution. Research into HVdc GIS is now spanning more than three decades. Past operational milestones include the installation of a gas insulated connection between a valve hall and a converter transformer in Gotland, Sweden in 1983 [8], a two year performance verification ending in 1999 run in the USA [9] and the successful operation of the Kii Channel HVdc link in Japan since 2000 [10]. The main focus of research into HVdc GIS during this time period has been on insulation characteristics of the gas volume, solid components and their interfaces [11]. Investigation of the switching operations by disconnectors and earthing switches integrated into these substations has received only minor attention during this period (cf. [12]-[14]).

\section{SCOPE OF RESEARCH}

With increasing complexity of HVdc multi-terminal grids and their substations, the need for switching cases other than fault current interruption will arise. Research into stateof-the-art switching equipment for $\mathrm{HVdc}$ by Cigré JWG A3/B4.34 [14] as well as a preliminary study of small-current switching cases within future $\mathrm{HVdc}$ substations by the authors [13] found bus-transfer switching to be a potentially relevant switching case. In double bus single breaker substations, a type widely used in ac transmission, bus-transfer switching by disconnector enables the reconfiguration of feeders within the substation while maintaining power flow without the need for $\mathrm{CB}$ switching. The authors have analyzed this ac switching case for a disconnector used in gas insulated switchgear (GIS) arriving at a reignition criterion which can be used to simulate bus-transfer in the time domain [15]. In the following, the same disconnector along with the theoretical findings is probed with respect to expected switching operations in future $\mathrm{HVdc}$ substations in order to identify unique challenges such as the lack of natural current zeroes and the harmonic content of the switching current. A different type of GIS disconnector using a far higher opening speed and air as an insulation medium was previously evaluated for its applicability in HVdc substations by Vinson [12] by means of constant current simulations. The study predicted a short arcing time well below comparable ac switching times for one, realistic application case.

It is important to note that there is a substantial difference in ac bus-transfer by disconnector in air insulated switchgear (AIS) compared to GIS. AIS is typically characterized by larger substation dimensions with higher self-inductance per unit length of conductor and disconnectors with considerable electrode travel in the hundreds to thousands of millimeters. For ac bus-transfer, this brings about a significantly higher 
recovery voltage, as is evident in the IEC standard 62271102 [16], which specifies recovery voltages between six to ten times larger for AIS substations of the the same rated voltage level. Peelo [17] concluded from measurements on air-break disconnectors and subsequent arc modelling, that bus-transfer for AIS relies on the negative differential arc resistance near current zero of sufficiently long switching arcs. Extinction occurs when the bus-transfer loop cannot sustain a further increase in arc voltage at decreasing switching current. In contrast, prior research by the authors [15] highlighted that negative differential arc resistance does not play a role in slow-moving GIS disconnectors, which exhibit electrode separation distances of less than $10 \mathrm{~mm}$ during relevant switching times. The arc voltage increases primarily with the separation of the electrodes, thus, making arc extinction certain only once the time-dependant arc voltage exceeds the recovery voltage at current zero. Therefore, the following derivations, measurements and simulations of bus-transfer processes apply exclusively to GIS disconnectors.

\section{Application of Limit Performance Prediction}

It has been shown previously, that for ac calculations, bustransfer processes can be simulated by using the equivalent circuit diagram shown in Fig. 1 (cf. [15], [18]). The influence of the switching arc of the disconnector is modelled as a voltage source $u_{\mathrm{DS}}$. The parallel sections of bus are modelled as inductances and resistances determined by the length of the bus and the technology of the substation (i.e. voltage level and insulation media). Therefore, $L_{1}$ and $L_{2}$ can be calculated from the inductance per unit length $L^{\prime}$ and the length of either bus $d_{1}$ or $d_{2}$. The same is true for $R_{1}$ and $R_{2}$ using the resistance per unit length $R^{\prime}$. For the investigated GIS designed for operation at a nominal voltage of $420 \mathrm{kV}$ ac, $L^{\prime}$ is approximately $200 \mathrm{nH} \mathrm{m}^{-1}$ and $R^{\prime}$ is $10 \mu \Omega \mathrm{m}^{-1}$ respectively. The influence of the resistances $R_{1}$ and $R_{2}$ are generally minor compared to the large impedances of the inductances $L_{1}$ and $L_{2}$ for typical ac substations. In early dc current commutation investigations by Kanngiesser and Cigré working group 14/14.08, the resistances were neglected for calculations of bus-transfer switching but considered in other commutation cases involving earth returns [19].

Before opening of the disconnector, the pre-switching current $i_{2}^{\mathrm{pre}}$ through the disconnector is determined by the lengths of the parallel bus $\left(d_{1}\right)$ as well as the total bus-transfer loop length

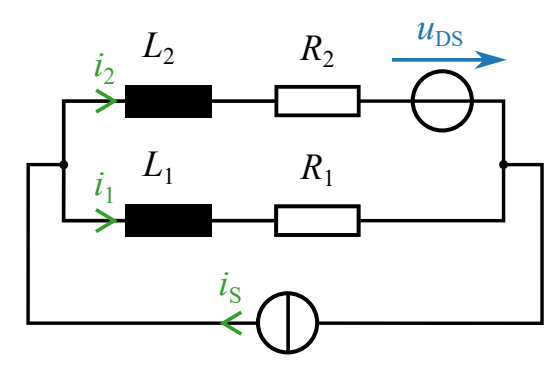

Fig. 1. Equivalent circuit diagram for bus-transfer breaking. The disconnector is modelled as a current and time dependant voltage source $\mathrm{u}_{\mathrm{DS}}$.
$\left(d_{\mathrm{BT}}=d_{1}+d_{2}\right)$ in combination with the total current $i_{\mathrm{S}}$ as follows:

$$
i_{2}^{\text {pre }}=\frac{d_{1}}{d_{\mathrm{BT}}} i_{\mathrm{S}}
$$

As soon as the disconnector opens, a switching arc develops, leading to the superposition of $i_{2}^{\text {pre }}$ with a counter current $i_{2}^{\text {arc }}$, which depends heavily on the arc voltage apparent at the disconnector terminals $\left(u_{\mathrm{DS}}\right)$. In the simplified case of a constant arc voltage $u_{\mathrm{DS}}(t)=u_{\mathrm{arc}}$, the counter current can be calculated analytically as

$$
i_{2}^{\operatorname{arc}}(t)=-\frac{u_{\text {arc }}}{R^{\prime} d_{\mathrm{BT}}}\left(1-e^{-\frac{\left(t-t_{i}\right)}{\tau}}\right),
$$

where $t_{i}$ refers to the time of ignition and $\tau$ is the timeconstant of the bus-transfer loop given by $L^{\prime} / R^{\prime}$. For currentor time-dependent arc voltage functions, analytical solutions for $i_{2}^{\text {arc }}(t)$ are rarely possible, making numerical calculations inevitable. However, for worst-case estimations, a constant arc voltage corresponding to the electrode fall voltage of a particular material can be used. In case of arcs in $\mathrm{SF}_{6}$ gas, $13.8 \mathrm{~V}$ and $17.5 \mathrm{~V}$ have been found as electrode fall voltages for tungsten and tungsten-copper electrodes respectively by Yokomizu [20].

Once the counter current reaches the level of the pre-switching current, the total current through the disconnector becomes zero. In previous investigations of a $420 \mathrm{kV}$ GIS disconnector opening at a speed of $0.1 \mathrm{~m} / \mathrm{s}$ in ac tests, the authors found, that when the current reached zero, conditions for thermal reignition were always present (cf. [15]). Therefore, extinction of the switching arc was certain when the recovery voltage

$$
u_{\mathrm{RV}}\left(t_{0}\right)=i_{\mathrm{S}}\left(t_{0}\right) R_{1}+\frac{d i_{\mathrm{S}}\left(t_{0}\right)}{d t} L_{1}
$$

was lower than the predicted arc voltage $u_{\mathrm{arc}}^{\mathrm{AC}}\left(t_{0}\right)$ at the time of current zero $t_{0}$ due to the circuit's inability to sustain an arc current. This was designated the extinction criterion.

The arc voltage for this particular type of disconnector was measured to increase with $a t^{b}$ from an initial voltage of $14.3 \mathrm{~V}$, where $a=235 \mathrm{Vs}^{-0.64}$ and $b=0.64$. Furthermore, the arc voltage was found to be virtually independent of current for the investigated arcing times. For $u_{\mathrm{RV}}\left(t_{0}\right)$ larger than $u_{\mathrm{arc}}^{\mathrm{AC}}\left(t_{0}\right)$, the likelihood of reignition increased towards higher recovery voltages but no clear criterion for reignition could be found. However, only very few extinctions were observed at recovery voltages equal to or greater than twice the expected arc voltage, thus a factor of two can serve as a rule of thumb for certain reignition.

In ac applications, $i_{\mathrm{S}}\left(t_{0}\right)$ and its time-derivative are linked by the grid frequency. Therefore, $u_{\mathrm{RV}}$ is often expressed as a combination of a length of bus $d_{1}$ and a source current $i_{S}$. For a specific type of bus, these two variables specify the recovery voltage completely according to eq. (3). With the addition of a pre-switching current or a current distribution between the two parallel buses, a bus-transfer switching case is fully characterized. As an example, the standard IEC-62271102 [16] currently specifies a recovery voltage of $20 \mathrm{~V}$ and a maximum pre-switching current of $1.6 \mathrm{kA}$ for $420 \mathrm{kV}$ GIS which corresponds to $d_{1}=160 \mathrm{~m}$ and pre-switching current of $80 \%$ of the total source current $i_{\mathrm{S}}=2 \mathrm{kA}$. 
In future dc grids, however, the source current is primarily a constant current determined by the dc power flow superimposed with a comparably small time-varying harmonic content. The derivative of this source current depends solely on the magnitudes, frequencies and relative phase angles of the harmonic currents. For this reason, $i_{2}^{\text {pre }}$ and $u_{\mathrm{RV}}$ are connected with an additional degree of freedom $\left(d i_{\mathrm{s}}\left(t_{0}\right) / d t\right)$. As a result, the process of reaching current zero is less strictly coupled to the likelihood of reignition compared to ac cases.

\section{FEASIBILITY PREDICTIONS FOR OPERATION IN DC}

Due to the lack of naturally occurring current zeros, it is prudent to investigate the time to initial current zero as a function of arc voltage, pre-switching current and bus-transfer loop length in order to evaluate the feasibility of bus-transfer in dc GIS. The results of a numerical simulation of these times are presented in Fig. 2 for the case of a constant dc source current distributed so that $i_{2}^{\text {pre }}$ is equal to $80 \%$ of $i_{\mathrm{S}}$. To identify the influence of the arcing voltage, the simulations were performed for a constant arc voltage of $13.8 \mathrm{~V}$ (cf. [20]) and for the arc voltage function previously found in ac test. Additionally, a modified ac arc characteristic corresponding to a contact separation speed of $0.3 \mathrm{~m} \mathrm{~s}^{-1}$ was simulated as well. It is evident that, for the considered types of arc voltages, both pre-switching current and bus-transfer loop length have very similar influences on the time to initial current zero in that their increase prolongs the time to initial current zero. It is important to note, that due to the exponential function in eq. (2), there is an absolute limit of bus-transfer loop length and pre-switching current for the case of constant arc voltage, representing an infinitely long arcing time. For the two other cases, no such limit exists as the arc voltages are strictly monotonically increasing with time. In general, the calculated times appear quite short. Only cases of high currents in combination with very long sections of bus require more than $10 \mathrm{~ms}$ to reach zero current when using the ac arc characteristic.

When evaluating the previously introduced extinction criterion for these simulated cases, the recovery voltage as defined in eq. (3) simplifies to $i_{\mathrm{S}} R_{1}$ due to the constant nature of the current. $R_{1}$ is typically very small in switchgear in order to minimize transmission losses and to prevent unnecessary resistive heating. At all times of initial current zero for the
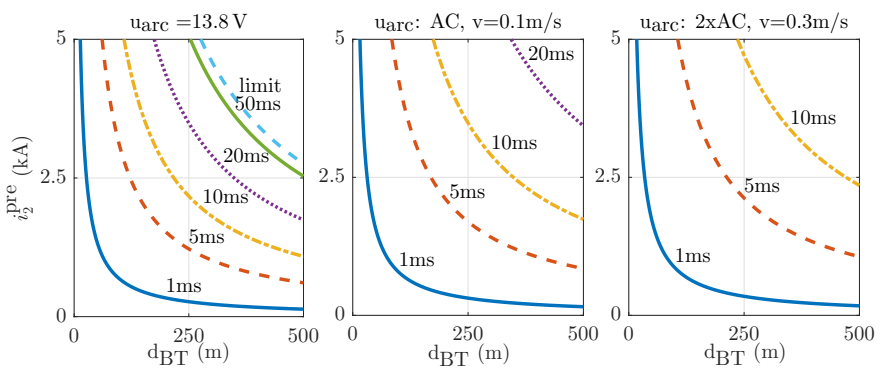

Fig. 2. Arcing times as functions of pre-switching current, total bus-transfer loop length and arc voltage. Left: constant arc voltage of $13.8 \mathrm{~V}$, middle: arc voltage characteristics found in ac investigations [15], right: ac arc voltage increasing twice as fast with respect to time, corresponding to an electrode separation speed of $0.3 \mathrm{~m} \mathrm{~s}^{-1}$ cases shown in Fig. 2, $u_{\mathrm{RV}}$ is smaller than the expected arc voltage. Therefore, no reignitions can occur, making the arcing time equal to time to initial current zero.

In reality, however, currents in HVdc applications are rarely constant in time. To analyze the influence of harmonic currents, Fig. 3 shows simulated cases of a source current of $2 \mathrm{kA}$ with a superimposed triangular current increasing and

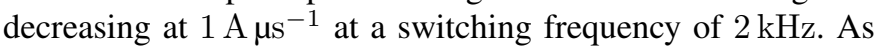
in the previous example, the pre-switching current is set to $80 \%$ of the source current. The total bus-transfer loop length is varied, so that the absolute value of the recovery voltage is either lower than, equal to or larger than the arcing voltage at time of ignition ( $d_{\mathrm{BT}}=50 \mathrm{~m}, 78 \mathrm{~m}$ and $170 \mathrm{~m}$ respectively). For these simulations, the extinction criterion is evaluated at each current zero. From the results of the constant arc voltage simulation presented in Fig. 3a, it is clear that the influence of the triangular source current onto the switching current increases with increasing bus-transfer loop length. For the shortest loop of $50 \mathrm{~m}$ the current gradient is negative during the entire arcing time. In contrast, the case of the intermediate loop of $78 \mathrm{~m}$ exhibits negative current gradients only when the source current is decreasing, otherwise $i_{2}$ stays nearly constant. The longest bus-transfer loop displays negative gradients when the source current is decreasing and positive gradients when the source current is increasing. The initial current zeros are reached at $1.5 \mathrm{~ms}, 2.7 \mathrm{~ms}$ and $4.8 \mathrm{~ms}$ respectively, at recovery voltages of $-7 \mathrm{~V},-12.7 \mathrm{~V}$ and $-24.3 \mathrm{~V}$. With the simulated arc voltage at a constant $13.8 \mathrm{~V}$, only the longest bus-transfer loop violates the extinction criterion at current zero, thus leading to a reignition in the simulation. The absolute values of the recovery voltages at all following current zeros are also greater than the arc voltage. Therefore, arc extinction never occurs despite the fact that the arc voltage is capable

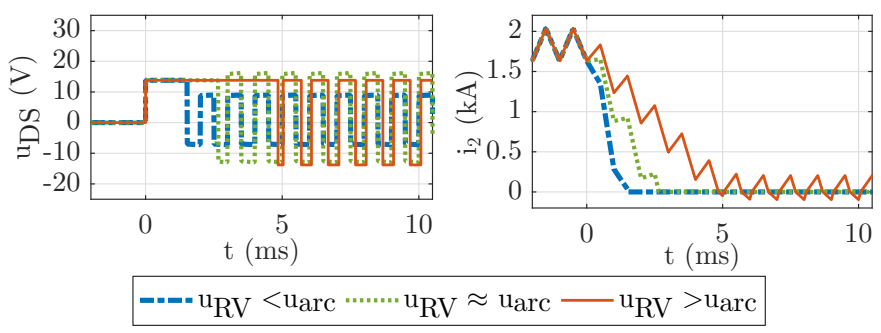

(a) $u_{\text {arc }}=13.8 \mathrm{~V}$

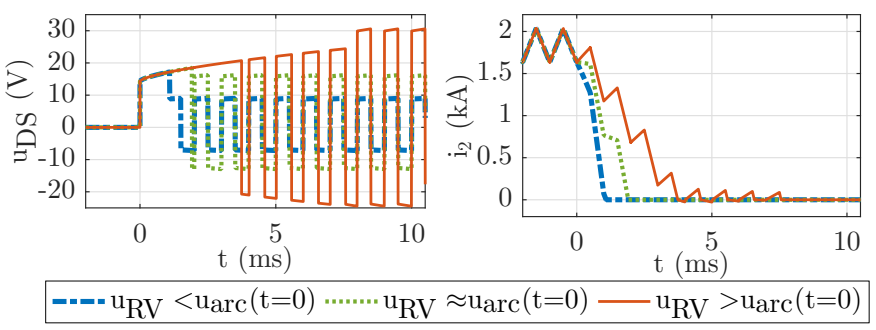

(b) $u_{\text {arc }}=u_{\text {arc }}^{\mathrm{AC}}$

Fig. 3. Disconnector voltage $u_{\mathrm{DS}}$ (left) and switching current $i_{2}$ (right) simulated for three different bus-transfer loop lengths corresponding to a recovery voltage smaller than the arc voltage $\left(d_{\mathrm{BT}}=50 \mathrm{~m}\right)$, a recovery voltage approximately the same as the arc voltage $\left(d_{\mathrm{BT}}=78 \mathrm{~m}\right)$ and a recovery voltage larger than the arc voltage at initial current zero $\left(d_{\mathrm{BT}}=170 \mathrm{~m}\right)$. 
of creating current zeros from the dc pre-switching current. In contrast, the simulation using the arc voltage function presented in Fig. $3 \mathrm{~b}$ shows an extinction of the switching arc regardless of the length of the bus-transfer loop. The current decrease is steeper in all cases leading to initial zeros at $1.1 \mathrm{~ms}$, $1.9 \mathrm{~ms}$ and $3.7 \mathrm{~ms}$. A total of eight reignitions are simulated to occur for the $170 \mathrm{~m}$ loop before the extinction criterion is finally fulfilled at an arcing time of $7.6 \mathrm{~ms}$ with an arc voltage of $-24.4 \mathrm{~V}$ and a recovery voltage of $-23.9 \mathrm{~V}$. It is clear from these simulation results that any recovery voltage is predicted to be surpassed by the arc voltage at some point as long as the disconnector is able to continue electrode separation and therefore continue to increase its arc voltage. However, the additional arcing time caused by the numerous reignitions needs to be taken into account in terms of electrode erosion and total arcing time. Furthermore, the applicability of the extinction criterion for such rapidly occurring current zeros as well as dc bus-transfer in general requires verification by testing.

\section{DC Bus-Transfer Test Setup}

To study bus-transfer operation in genuine dc operations, a test setup as shown in the equivalent circuit diagram of Fig. 4 was devised. Having previously proved its merit for bus-transfer testing in an ac setup (cf. [15]), the bus-transfer loop including the disconnector and its control unit were modified as little as possible in order to assure a high level of comparability. The current source, however, was replaced entirely since prior investigations relied on an LC-oscillating current source capable solely of providing ac. The setup presented in Fig. 4 contains three buck converter modules $\left(M_{1}\right.$,

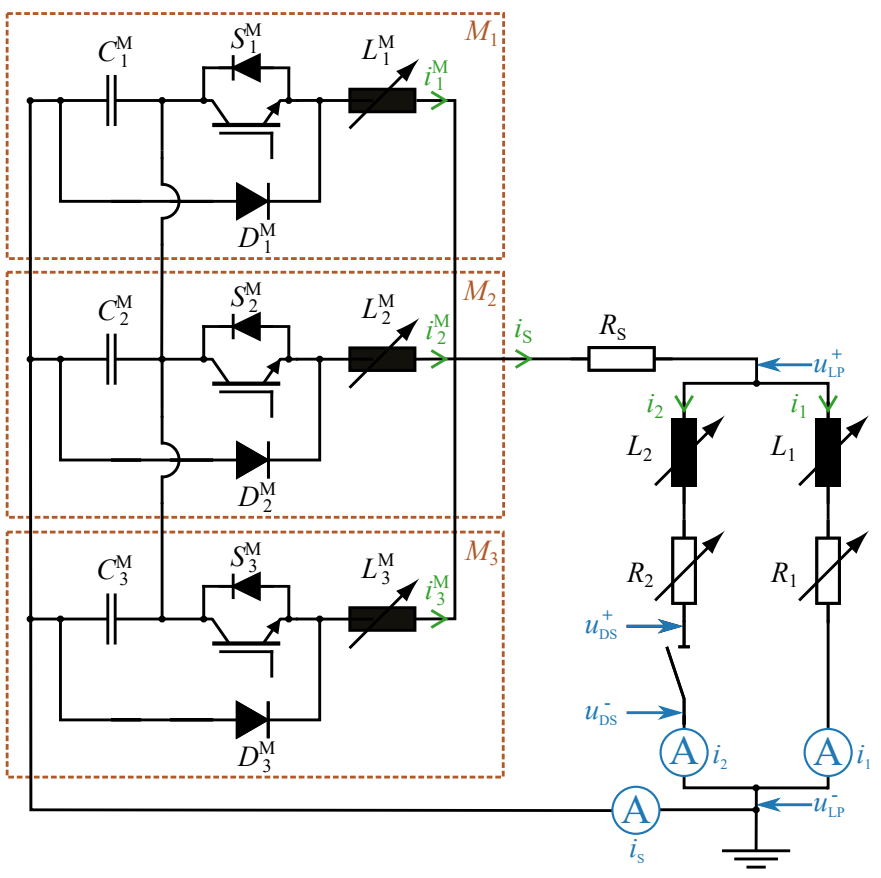

Fig. 4. Equivalent circuit diagram of the flexible pulsed dc source connected to the bus-transfer loop through an additional load resistor.
TABLE I

COMPONENT VALUES OF THE PARTS USED IN THE DC BUS-TRANSFER TEST SETUP.

\begin{tabular}{ll}
\hline \hline Component & Characteristics \\
\hline$C_{1 \ldots 3}^{\mathrm{M}}$ & $2 \mathrm{mF}, 0 \mathrm{kV} \ldots 3 \mathrm{kV}$ \\
$L_{1 \ldots 3}^{\mathrm{M}}$ & $6 \mu \mathrm{H} \ldots 4500 \mu \mathrm{H}$ \\
$S_{1 \ldots 3}^{\mathrm{M}}$ & low loss IGBT, $1.2 \mathrm{kA}, 4.5 \mathrm{kV}$ \\
$D_{1 \ldots 3}^{\mathrm{M}}$ & low loss diode $1.3 \mathrm{kA}, 4.5 \mathrm{kV}$ \\
$R_{\mathrm{S}}$ & $0.08 \Omega \ldots 2 \Omega$ \\
$L_{1}, L_{2}$ & $6 \mu \mathrm{H} \ldots 275 \mu \mathrm{H}$ \\
$R_{1}, R_{2}$ & $0.4 \mathrm{~m} \Omega \ldots 18 \mathrm{~m} \Omega$ \\
$u_{\mathrm{DS}}^{+}, u_{\mathrm{DS}}^{-}, u_{\mathrm{LP}}^{+}, u_{\mathrm{LP}}^{-}$ & passive voltage probes, $100: 1,400 \mathrm{MHz}$ \\
$i_{1}, i_{2}, i_{\mathrm{S}}$ & HOKA current transducers, $0 \mathrm{~Hz} . .1 \mathrm{MHz}$ \\
\hline \hline
\end{tabular}

$M_{2}, M_{3}$ ) connected in parallel to deliver a total dc current of up to $3 \mathrm{kA}$ to the bus-transfer loop. The intricacies of this flexible pulsed dc source (FPDCS) have recently been presented by the authors [21]. Its main features include individually variable output inductances $\left(L_{1}^{\mathrm{M}}, L_{2}^{\mathrm{M}}, L_{3}^{\mathrm{M}}\right)$ which allow current gradients of up to $50 \mathrm{kA} \mathrm{ms}^{-1}$, a maximum energy of $27 \mathrm{~kJ}$ per test from a set of three parallel capacitors $\left(C_{1}^{\mathrm{M}}, C_{2}^{\mathrm{M}}, C_{3}^{\mathrm{M}}\right)$ and individually controllable power semiconductors $\left(S_{1}^{\mathrm{M}}, S_{2}^{\mathrm{M}}\right.$, $S_{3}^{\mathrm{M}}$ ) capable of switching frequencies between $0.1 \mathrm{kHz}$ to $10 \mathrm{kHz}$. An overview of all hardware components and their characteristic values is shown in Table I. The main limitations in terms of safe operating area are given by the energy storage of the capacitors, the heating of the power semiconductors and the parasitic properties of both source and load (i.e. selfinductances of wires and parasitic capacitances to ground). For loads exhibiting low voltages compared to the capacitor voltage of the source, an additional load resistor $R_{\mathrm{S}}$ can be connected in series to the load. This is especially useful if the desired source current $i_{\mathrm{S}}$ is to have symmetric harmonic components. The currents produced by this setup are similar to the simulated $i_{\mathrm{S}}$ of Fig. 3. While these currents are not an accurate representation of realistic dc currents overlaid with harmonics of certain frequencies, they are differentiable with a high numerical accuracy. Therefore, analysis of recovery voltages at interesting points in time, such as current zeroes, is greatly facilitated compared to more complex currents. The knowledge gained analyzing tests using these artificial currents then can be applied to realistic dc currents in simulation. Two source modules were utilized in parallel to produce currents similar to the symmetric ones shown in Fig. 3. Module $M_{2}$ was configured with a large inductance $L_{2}^{\mathrm{M}}$ of $4.5 \mathrm{mH}$, so as to deliver an almost constant dc current offset. The inductance of $M_{1}$ was set to $0.5 \mathrm{mH}$ or $1.5 \mathrm{mH}$ depending on the test to superimpose a fast-changing triangular current onto $i_{2}^{\mathrm{M}}$. In this configuration, six different operating states are possible as listed in Table II. Special attention has to be paid to the two operating states during which $i_{1}^{\mathrm{M}}=0$. In these states, the current gradient is determined by $L_{2}^{\mathrm{M}}$, which is between three and nine times larger than $L_{1}^{\mathrm{M}}$. Therefore, $u_{\mathrm{RV}}$ during these periods is reduced by a factor of three to nine compared to the remaining four switching states, which can influence both switching current and reignition behavior as predicted by the 
TABLE II

INFLUENCE OF THE SWITCHING STATES OF $S_{1}^{\mathrm{M}}$ AND $S_{2}^{\mathrm{M}}$ ONTO THE TOTAL SOURCE CURRENT $i_{\mathrm{S}}$ AND THE RECOVERY VOLTAGE $u_{\mathrm{RV}}$.

\begin{tabular}{cccc}
\hline \hline$S_{1}^{\mathrm{M}}$ & $S_{2}^{\mathrm{M}}$ & $i_{\mathrm{S}}$ & $\frac{d i_{\mathrm{s}}}{d t}, u_{\mathrm{RV}}$ \\
\hline on & on & increasing, fast & positive, high \\
off & on & decreasing, fast & negative, high \\
off, $i_{1}^{\mathrm{M}}=0$ & on & increasing, slow & positive, low \\
on & off & increasing, fast & positive, high \\
off & off & decreasing, fast & negative, high \\
off, $i_{1}^{\mathrm{M}}=0$ & off & decreasing, slow & negative, low \\
\hline \hline
\end{tabular}

simulation results shown in Fig. 3.

The bus-transfer loop consists of two parallel connected, purpose-built GIS bus equivalents constructed of wound aluminium conductor, each of which can be configured to represent anywhere between a few meters of bus up to a maximum of $600 \mathrm{~m}$. The resistance per emulated unit length $R^{\prime}$ is aimed to be approximately $10 \mu \Omega \mathrm{m}^{-1}$ and the inductance $L^{\prime}$ in the range of $200 \mathrm{nH} \mathrm{m}^{-1}$. From the simulation results shown in Fig. 3 using the same numerical values for inductance and resistances, it is expected that the recovery voltage across the open disconnector is dominated by the inductive voltage drop with the resistive part playing a minor role. Therefore, the recovery voltage $u_{\mathrm{RV}}$ is expected to be approximately proportional to $d i_{\mathrm{S}} / d t$ as listed for each switching state in Tab. II. During testing, it was found that certain combinations of bus-transfer loop settings and source impedances presented resonances with frequencies in the hundreds of kilohertz, which are excited by switching of $S_{1}^{\mathrm{M}}$ and $S_{2}^{\mathrm{M}}$. Especially switching $S_{1}^{\mathrm{M}}$ with open disconnector provoked measurable transient recovery voltage overshoots due to the jump between high positive and high negative recovery voltage across the disconnector within microseconds. With the disconnector in closed position and while switching, oscillatory voltage components were generally immeasurable. Since the studied extinction criterion is based on the assumption of thermal reignition, $u_{\mathrm{RV}}$ generally refers to the recovery voltage without transient overshoot in the following, except when stated otherwise (i.e. in cases when the transient overshoots are of relevance).

The seven measurement devices shown in Fig. 4 were deployed to achieve as high a measurement quality as possible. Four potentials were measured using high-impedance, highfrequency voltage dividers referenced to the common ground shown in the diagram. The voltage drop across the disconnector was calculated in post-processing from $u_{\mathrm{DS}}^{+}$and $u_{\mathrm{DS}}^{-}$while at the same time removing the influence of the inductance of the pieces of GIS separating the two voltage dividers. $u_{\mathrm{LP}}^{+}$and $u_{\mathrm{LP}}^{-}$were used in an identical fashion to calculate the total voltage drop across the bus-transfer loop $u_{\mathrm{LP}}$. Additionally, they were employed as automatic error detections by analyzing the difference of $u_{\mathrm{LP}}^{-}$to ground potential to detect ground failures and by comparing $u_{\mathrm{LP}}$ to $u_{\mathrm{DS}}$ after switching arc extinction to verify the measurement and calculation of the disconnector voltage. The currents $i_{1}, i_{2}$ and $i_{\mathrm{S}}$ were measured using identical, purpose built current transducers according to the HOKA principle (cf. [22]). The current sensors operate electrically isolated, providing $1 \%$ measurement accuracy over a current range of $0 \mathrm{kA}$ to $3 \mathrm{kA}$ from pure dc up to a maximum frequency of $1 \mathrm{MHz}$. In post-processing, the sum of $i_{1}$ and $i_{2}$ was compared to $i_{\mathrm{S}}$ to verify the correct operation of all current transducers.

\section{Measurement Analysis and Evaluation}

The post-processing of the measured currents and voltages has proven to require an extensive set of software tools. In an automated first step, the differential signals $u_{\text {DS }}$ and $u_{\mathrm{LP}}$ are calculated and all raw data are downsampled to a sampling frequency of $1 \mathrm{MS} \mathrm{s}^{-1}$. Using the time-derivative of the downsampled $i_{2}, u_{\mathrm{DS}}$ is compensated for the inductive voltage drop between the two relevant voltage dividers. All other measured signals remain unchanged.

In order to characterize the pre-switching current distribution of $i_{\mathrm{S}}$ into $i_{1}^{\mathrm{pre}}$ and $i_{2}^{\mathrm{pre}}$, tests are performed during which the disconnector stays in its closed position. This effectively shortcircuits the device under test, the results are thus referred to as short-circuit (SC) data. Similarly, open-circuit (OC) tests take place with the disconnector remaining open, so that the recovery voltage is apparent at the disconnector poles during the entire duration of the test. A series of measurements started with changing of the bus-transfer inductances $L_{1}$ and $L_{2}$, which necessitated the recording of SC and OC measurements. Bus-transfer tests were performed at different intervals ranging from minutes to days in order to minimize potential influences of the test sequence.
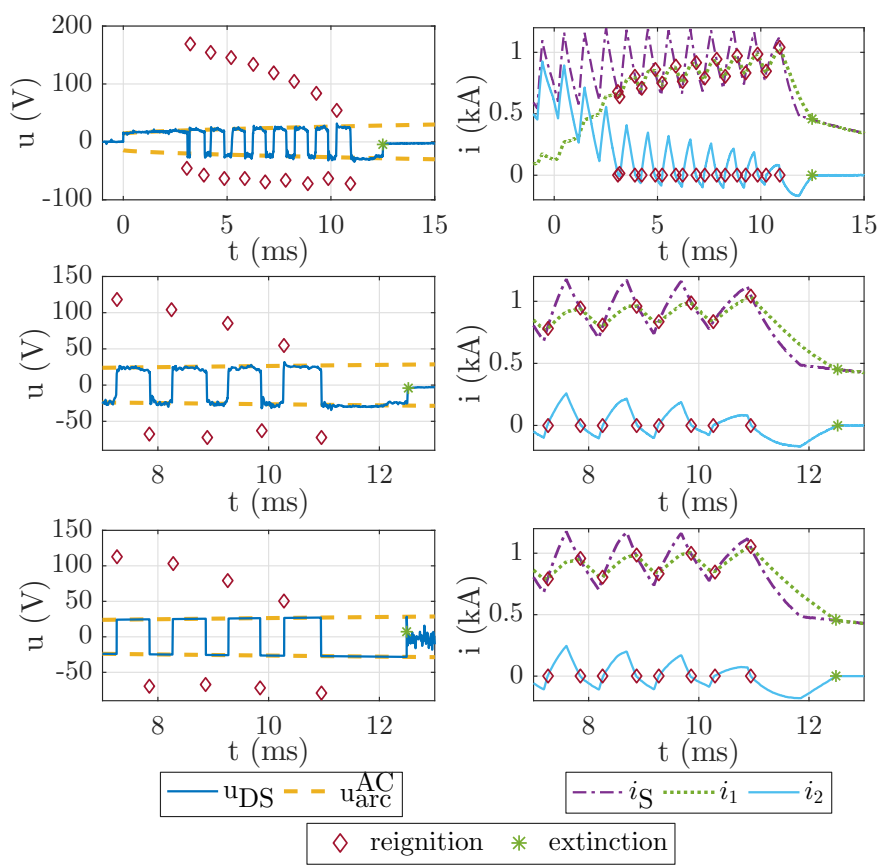

Fig. 5. Example evaluation results of a test exhibiting 17 reignitions in terms of disconnector voltage (left) and bus-transfer currents (right); Top: Entire measurement time from arc ignition to extinction. Middle: zoom to final nine current zeros; Bottom: Simulation based on ac arc characteristic and measured $i_{\mathrm{S}}$. Locations of the reignition and extinction markers indicate calculated $u_{\mathrm{RV}}$ (left) and measured currents (right) at current zero. 
The differential equations describing the equivalent circuitdiagram of the source and bus-transfer loop shown in Fig. 4 have been analyzed for SC and OC tests for all six switching states listed in Tab. II. With small error, the source current for a particular test case and point in time can be described as

$$
i_{\mathrm{S}}(t) \approx \gamma\left(1-e^{-\delta\left(t-t_{0}\right)}\right)+i_{\mathrm{S}}\left(t_{0}\right)
$$

where $t_{0}$ refers to the time of the most recent change in switching state. However, for all analyzed cases modelling $i_{\mathrm{S}}$ as a second order polynomial approximation around $t_{0}$ resulted in more consistent and accurate results, thus $i_{\mathrm{S}}$ was fitted using the expression

$$
i_{\mathrm{S}}(t)-i_{\mathrm{S}}\left(t_{0}\right) \approx \alpha\left(t-t_{0}\right)-\beta\left(t-t_{0}\right)^{2},
$$

where $\alpha$ and $\beta$ are fitting parameters depending on the configuration of the test setup and the energy stored in the capacitors at $t_{0}$. Fitting eq. (5) onto the measured current has proven to be the most reliable method to determine the recovery voltage at any point in time because it smooths measurement noise while enabling the analytical derivation of $i_{\mathrm{S}}$ necessary to determine $u_{\mathrm{RV}}$ at specific points such as current zeros. In parallel to this calculation applicable only for specific points in time selected by the user, a simulation spanning the entire time of a test is performed automatically. As inputs for this simulation, the measured $i_{\mathrm{S}}$ as well as $L_{1}, L_{2}, R_{1}$ and $R_{2}$ determined during SC and OC measurements are combined with the arc characteristic and the extinction criterion of the prior ac investigations. The aim of this simulation is to test the applicability of the limit performance prediction method using minimal user input.

In Fig. 5, an example of the evaluation results of a bus-transfer test are presented. For each of the 18 current zeros which occur during this test, a section of time has to be selected for $u_{\mathrm{RV}}$ calculation by fitting $\alpha$ and $\beta$ onto $i_{\mathrm{S}}$ as specified by eq. (5). Furthermore, the user selects the exact time of current zero and its classification (i.e. reignition, extinction, special case), which automatically saves the calculated values of $u_{\mathrm{RV}}$ at these points in time and prints markers at the appropriate voltages. An average of $u_{\mathrm{DS}}$ over $1 \mathrm{~ms}$ leading up to every userselected current zero is saved as arc voltage associated with this point in time for comparison to the calculated recovery voltage. The aforementioned automatic simulation of the test based on the ac extinction criterion is shown for the final $5.5 \mathrm{~ms}$ of the test as well. It exhibits good agreement with the manually evaluated results with the exception of the time after arc extinction when the small-amplitude, high-frequency measurement noise of $i_{\mathrm{S}}$ provokes erroneously high recovery voltages.

\section{Example Validation Measurements}

In order to understand the applicability and the implications of the aforementioned extinction criterion for dc tests, a bustransfer test was set up which exhibited a constant recovery voltage close to the anticipated arc voltage. This was realized by a long and steady initial rise of the source current during which arc ignition took place. During this rise, the anticipated recovery voltage can be considered almost constant with $L_{1}$ chosen so that $u_{\mathrm{RV}}$ is very close to the anticipated arc voltage. After the initial rise, the source current would fall very slowly to produce recovery voltage below $1 \mathrm{~V}$. From the simulations based purely on the ac results presented in Fig. 3 it is expected that the switching current remains constant when $u_{\mathrm{RV}} \approx u_{\mathrm{arc}}^{\mathrm{AC}}$ during the initial rise but that it goes to zero rapidly when $u_{\mathrm{RV}} \ll u_{\mathrm{arc}}^{\mathrm{AC}}$ afterwards. The results of three example tests are shown in Fig. 6. In example (a), the arc voltage consistently measured a few volts higher than anticipated arc voltage (and therefore the recovery voltage). As a result, the initial switching current of $60 \mathrm{~A}$ decreases to zero slowly over a time of $1.4 \mathrm{~ms}$. At which point, the calculated recovery voltage is $15.7 \mathrm{~V}$, which is lower than the expected arc voltage of $17.7 \mathrm{~V}$. As expected for such a case, no reignition occurs. In examples (b) and (c), the arc voltages follow the predictions rather well, leading to almost constant $i_{2}$ during rising currents. In example (b) current zero is reached during the first decrease of $i_{\mathrm{S}}$ at a calculated recovery voltage of less than $0.1 \mathrm{~V}$. However, in example (c), the duration of the source current decrease is too short to allow $i_{2}$ to reach zero, leading to a second (short) period of constant current before the final decrease to zero. From these three examples in combination with the evaluated example shown in Fig. 5, it is evident that the assumptions made based on the ac limit performance prediction hold for many of the analyzed tests. Namely, the development of the arc voltage as a function of time, the applicability of the extinction criterion and the validity of the switching current calculation have been verified for dc bus-transfer tests.

Over the course of evaluating a total of 226 bus-transfer tests exhibiting a combined 1070 current zeros, six instances of very similar but unexpected behavior were also identified. The evaluation of one of these tests is shown in Fig. 7. The test setup was the same as used for the measurement shown in Fig. 5 but due to jitter in the motor control of the disconnector, the start of the arcing time is different. Therefore, $i_{2}^{\text {pre }}$ differs by $90 \mathrm{~A}$ and with it the time to initial current zero by $0.1 \mathrm{~ms}$. The unpredicted behavior occurs immediately after the initial current zero at an arcing time of $2.97 \mathrm{~ms}$. The calculated recovery voltage is $-44.2 \mathrm{~V}$, the predicted
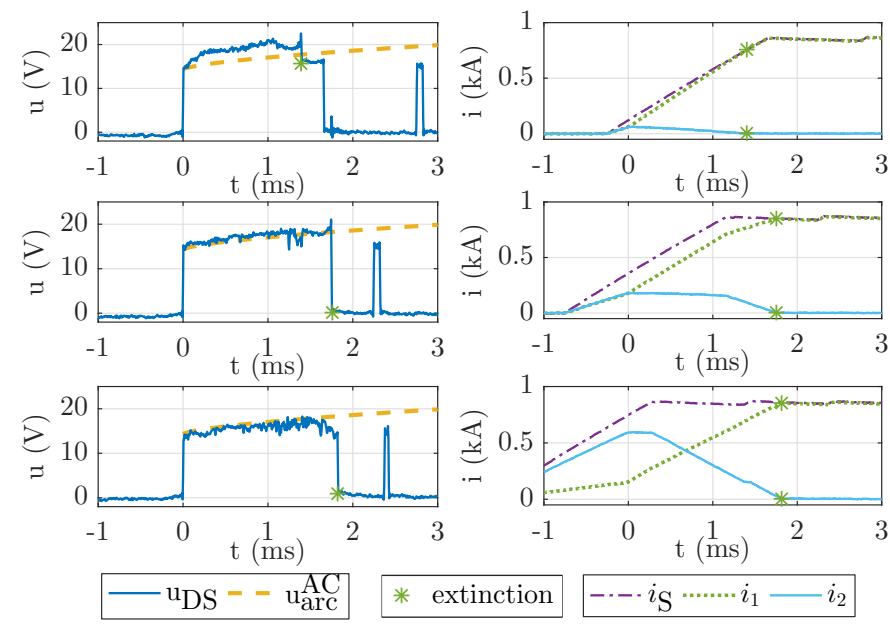

Fig. 6. Three example measurements of note exhibiting no reignitions. 
arc voltage $-19.8 \mathrm{~V}$. Therefore, the extinction criterion is not fulfilled implying that a reignition at this point is possible but not certain. From the magnification around this point shown in the figure, it can be seen that $i_{2}$ stays zero (within measurement accuracy) while a voltage varying between $-31.9 \mathrm{~V}$ and $-41.1 \mathrm{~V}$ is apparent at the disconnector. The match between this measured voltage and the calculated recovery voltage implies that an extinction of the switching arc took place at the initial current zero. However, $48 \mu$ s later, a change in switching state of the current source causes a fast rise of the measured recovery voltage to $172 \mathrm{~V}$. After a short transition period of $4 \mu \mathrm{s}$, the disconnector voltage settles close to the predicted arc voltage at around $20 \mathrm{~V}$ and $i_{2}$ starts to increase as after a normal reignition. Due to the short delay between initial current zero and reignition, this phenomenon is referred to as delayed reignition in this context. From the limited set of data, it is impossible to ascertain the mechanism of reignition. Time scales for thermal reignition in well-designed $\mathrm{SF}_{6}$ circuit breakers are typically in the range of a few microseconds [23]. However, the transition regime between thermal and dielectric breakdown (referred to as Paschen by Schoetzau) regimes during which temperatures above $1500 \mathrm{~K}$ exist has been found to last up to $200 \mu$ s for a model double flow circuit breaker in $\mathrm{SF}_{6}$ by Schoetzau [24]. It is likely that this time scale extends considerably in case of an unblown, free burning arc in a very small electrode gap (i.e. less than $1 \mathrm{~mm}$ for all recorded cases) due to a lack of gas exchange as is the case in the presented disconnector. Additionally, the short arc is likely to contain high levels of copper contamination from the electrodes which increases the conductivity of the hot gas during the thermal and transition regime considerably, increasing the likelihood of thermal reignition. For a $5 \%$ copper contamination of $\mathrm{SF}_{6}$ at $2800 \mathrm{~K}$ a conductivity increase by three orders of magnitude was found by Mentel [25]. Once the gas between the electrodes reaches temperatures of the Paschen regime, reignition
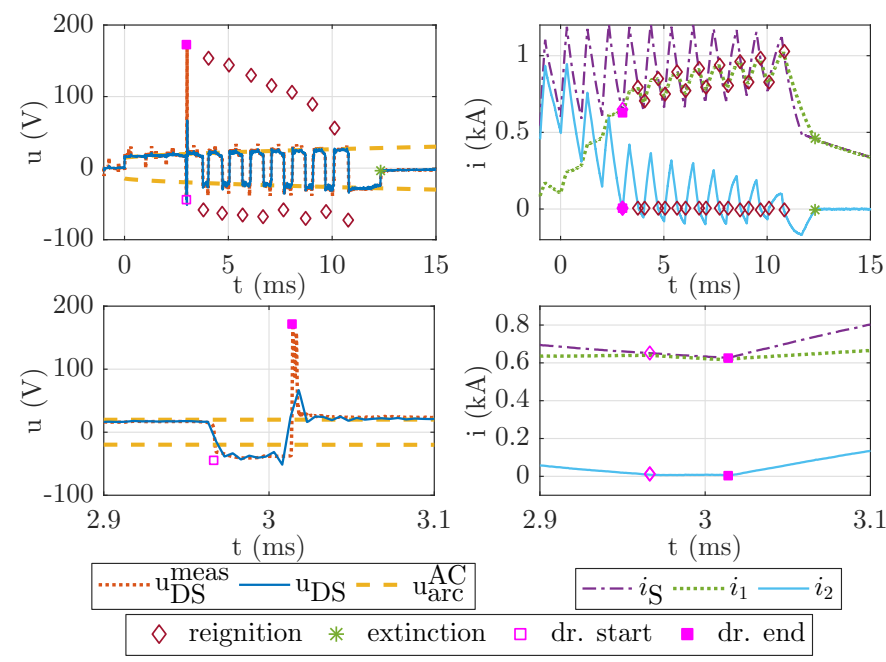

Fig. 7. Example of a measurement exhibiting a delayed reignition at the initial current zero. Top: Overview of the whole bus-transfer. Bottom: Magnification of $0.2 \mathrm{~ms}$ surrounding the delayed reignition. Disconnector voltage (left) contains high resolution, uncompensated measurement signal $\mathrm{u}_{\mathrm{DS}}^{\text {meas }}$ in addition to inductance-compensated and downsampled $\mathrm{u}_{\mathrm{DS}}$. by transient switching overvoltages due to the substantially lowered critical field strength of hot mixtures of $\mathrm{SF}_{6}$ and copper becomes a possibility. As shown by Mentel [25], the ionization potential of copper contaminated $\mathrm{SF}_{6}$ is lower than that of pure $\mathrm{SF}_{6}$, thus lowering electric strength of the gas gap. For the presented example, the electric field shortly before reignition is within the range of critical fields calculated by Wang and Yao. [26], [27] for a $50 \% \mathrm{SF}_{6}-50 \% \mathrm{Cu}$ gas mixture of $1000 \mathrm{~K}$. However, one of the six recorded instances of delayed reignition occurred at a time of no switching action of the source at a level of recovery voltage below what is necessary for dielectric breakdown even of contaminated hot $\mathrm{SF}_{6}$, thus lending more credibility to thermal reignition.

It is important to note that, for the recorded instances of delayed reignition, the calculated recovery voltage and the measured disconnector voltage at time of current zero were higher than the predicted arc voltage. When applying the extinction criterion for limit performance prediction, these current zeros would, therefore, be simulated as instantaneous reignitions which would result in an accurate worst-case calculations. In prior ac investigations, no reignitions were recorded after periods of zero current of any length, which led to the assumption that the conditions necessary for reignition decayed very quickly. However, in said ac tests the recovery voltage was a comparably slow changing sinusoidal as opposed to the step changes of large amplitude apparent in the presented dc tests. In order to further study the criteria of reignition, the test setup of Fig. 4 would need to be extended considerably, such as by installing a post-arc current measurement system able to measure small currents during the thermal phase and devising a method to determine the composition of the arc plasma. Using such a setup, measurements aiming to apply specific voltage stress in different time steps after current zero could be used to better define the different phases of recovery for this type of GIS disconnector.

\section{Synopsis Of EVAluATED Data}

In addition to the examples presented above, a further 221 bus-transfer tests were conducted varying both pre-switching current and recovery voltage. In Fig. 8 the recovery voltages for all current zeros (extinctions, immediate reignitions and delayed reignitions) below $100 \mathrm{~V}$ are plotted against the time to current zero. Additionally, the measured arc voltages leading up to each current zero are given together with the expected arc voltage development from prior ac tests using the same disconnector. In these results, a clear divide between recovery voltages leading to reignitions and those leading to extinctions is evident. As was expected, no reignition occurred at a recovery voltage below the arc voltage for a given arcing time. Thus, the extinction criterion can be considered experimentally validated for dc bus-transfer.

In a time range of $4 \mathrm{~ms}$ to $6 \mathrm{~ms}$, recovery voltages close to the arcing voltages have been achieved. Extinctions, immediate reignitions and one delayed reignition at recovery voltages of $23.5 \mathrm{~V}$ to $36.1 \mathrm{~V}$ were measured. However, all current zeros followed by recovery voltages above $40 \mathrm{~V}$ have been recorded as either immediate or delayed reignitions for times 


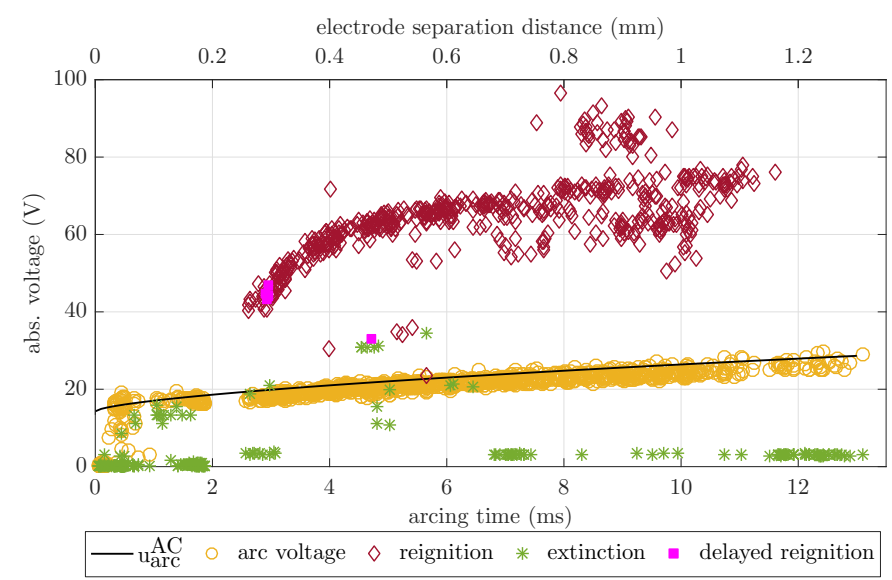

Fig. 8. Summarized results of 1070 current zeros which occurred at varying recovery voltages cropped to $0 \mathrm{~V}$ to $100 \mathrm{~V}$ as exclusively immediate reignitions occur above $100 \mathrm{~V}$. Arc voltages are averaged starting at $1 \mathrm{~ms}$ before current zero. Recovery voltages for reignition and extinction calculated at current zero. For delayed reignitions, only the calculated recovery voltages at the time of reignition are shown.

to current zero between $2.6 \mathrm{~ms}$ to $11.6 \mathrm{~ms}$. It is thus reasonable to infer, that recovery voltages of two to three times the level of the predicted arc voltage always lead to reignitions within these times. For the intermediate region between guaranteed extinction and a recovery voltage double the arc voltage, not enough data is available yet to draw a conclusion.

With this validation of the extinction criterion for dc bustransfer, the applicability of limit performance estimation by time-based simulation as presented above in Figs. 2 and 3 is given as well. The results of the automated simulation of each test generally agree very well with the measured results. The exceptions are current zeros exhibiting recovery voltages in the region between arc voltage and certain reignition. When applying the extinction criterion, such current zeros are always treated as reignitions in order to simulate a worst-case limit performance. In reality, however, this band of recovery voltages shows both reignitions and extinctions. A secondary source of mismatch between measurement and simulation stems from the fact that the switching arc presents some stochastic behavior in its voltage over time (cf. examples in Fig. 6). Especially when combined with the sharp transitions in source current gradient, these deviations from the expected

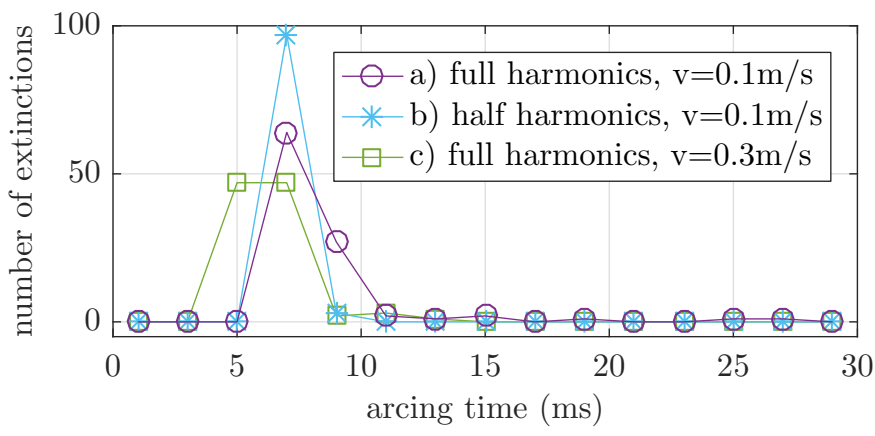

Fig. 9. Simulation of 100 bus-transfers for three cases of different harmonic magnitudes and electrode separation speeds. arc voltage can lead to misestimations of the arcing time. Using the simulations to predict switching performance over a sufficiently large number of samples, however, these errors should become negligible.

In order to conduct such simulations employing the extinction criterion, precise data on the expected current harmonics are needed together with the layout and technology of the substation in which the disconnector will be located. While many studies of ac side harmonics of $\mathrm{HVdc}$ converters exist due to the interactions of dc links with ac grids (cf. [28]), not much data is available on dc side harmonics, especially for the case of future multi-terminal networks. According to IEC/TR62543 [29] and Cigré Technical Brochure 269 [30] numerous combined factors determine the harmonic voltage and current of a VSC converter such as converter technology (number of levels, cell capacitor size, etc.), dc side filters, switching frequency, modulation method, operating conditions and ac side operation. For multi-terminal operation the interaction between converters, the switching states of the substations and the grid topology are likely to play an additional role.

In Fig. 9 results of such dc bus-transfer simulations are shown using a simple source current

$$
i_{\mathrm{S}}(t)=i_{0}+\sum_{n=1}^{10} \alpha_{n} \sin \left(n \omega_{0} t+\varphi_{n}\right),
$$

where the dc current $i_{0}$ is set to $2 \mathrm{kA}$, the lowest harmonic frequency considered $\omega_{0}$ is chosen as $1 \mathrm{kHz}$ and the harmonic current magnitudes are set to $\alpha_{n}=(55-5 n) \mathrm{A}$, so as to emulate harmonic contents closer to the ones likely to be found in future multi-terminal dc grids compared to the artificial test currents above. This current is split into a bus-transfer loop of $d_{1}=160 \mathrm{~m}$ and $d_{2}=40 \mathrm{~m}$. Three different scenarios have been simulated 100 times each, randomly selecting the phase angles $\varphi_{n}$ and the ignition time of the switching arc. In scenario a), the extinction criterion has been used, assuming the disconnector characterized in the measurements presented above. It is evident from the results, that the majority of simulated arcing times are within $6 \mathrm{~ms}$ to $10 \mathrm{~ms}$. However, in nine simulations out of 100 , the arcing time is more than $10 \mathrm{~ms}$. The most extreme case simulated exhibits 277 reignitions and a total arcing time of $41.6 \mathrm{~ms}$. According to the extinction criterion, there exist two possible ways to mitigate the occurrence of such long arcing times; Either the recovery voltage is reduced or the arc voltage is increased at current zero. In scenario b), the grid-side possibility of reducing the harmonic current magnitude by half is simulated. Such a change could be the result of increased high frequency filtering or an alteration of the converter modulation scheme, for example. As a result of this change, the maximum arcing time is reduced to $8.1 \mathrm{~ms}$ and the maximum number of reignitions becomes six, denoting a significant reduction comparing to the case of full harmonic content. The second possibility of arcing time reduction is demonstrated in scenario c), where the recovery voltage is the same as scenario a) but the arc voltage increases twice as fast, which corresponds to an electrode separation speed of $0.1 \mathrm{~m} \mathrm{~s}^{-1}$. In 92 out of the 100 simulated cases arcing times between $5 \mathrm{~ms}$ to $7 \mathrm{~ms}$ are recorded while 
no arcing time longer than $12.5 \mathrm{~ms}$ was found. However, the maximum arcing time also corresponds to a switching case of 52 reignitions at current zero.

\section{IMPLICATIONS FOR FUTURE HVDC APPLICATION}

A number of implications for development, testing and application of disconnectors for bus-transfer switching arise from the presented results:

Prediction of the bus-transfer process in terms of switching current, recovery voltage and switching arc extinction is possible using a simple model of the arc voltage behavior over time and the presented estimation criterion as long as the arc voltage remains reasonably independent of the switching current.

Application of existing ac disconnectors with bus-transfer capability in future gas insulated $\mathrm{HVdc}$ substations is feasible in terms of switching performance. Simulations and measurements have shown that current zeros can be reached within reasonable arcing times, even for large substations and high pre-switching currents.

Harmonic content of the bus-transfer current plays a substantial role in the delay of current zero and in the reignition of the switching arc at current zero. It is, therefore, important to know the maximum harmonic content of the current in an $\mathrm{HVdc}$ substation in terms of magnitudes, frequencies and phase angles to predict bus-transfer performance of disconnectors. For widespread application of disconnectors, a standardization of the maximum harmonic currents present in dc substations is therefore necessary.

Simulation of the bus-transfer performance of a given disconnector is achievable as soon as its arcing behavior is known and the application is well-defined especially in terms of bus lengths and harmonic content of the currents. In order to obtain a prediction of the limit performance of a given disconnector, repeated simulations accounting for small variations in arc voltage and harmonic currents are necessary (i.a. Monte Carlo simulations).

Modifications of the disconnector which directly influence the arc voltage behavior, such as changes in electrode separation speed, can be assessed in simulation by using the limit performance prediction reducing the need for development tests. The same is true for changes in the grid-side requirements, which affect the pre-switching current and recovery voltage. Testing of bus-transfer switching (and similar commutation operations) is practicable using a circuit such as the proposed full bus-transfer loop in combination with a dc source. The dc source needs to be able to sustain a high current during load change while simultaneously generating a harmonic spectrum similar to what will be found in application.

Reignitions caused by high frequency harmonic currents are likely to be of considerably higher repetition rate and lower peak current compared to known ac applications. Further research is necessary to clarify if existing erosion models, such as the one developed by Tepper et al. [31] for copper-tungsten contacts in circuit breakers, are capable of estimating the mass loss caused by this particular switching case. Moreover, the delayed reignitions recorded for a small number of current zeros should be investigated further in order to advance the extinction criterion with regard to fast changes of the recovery voltage immediately following current zero.

Extension of disconnector applications to other switching duties similar to bus-transfer in multi-terminal HVdc grids is conceivable. By employing the presented simulations, limit performance of a similar disconnector for such switching cases can be evaluated with relative ease. Transferring lines under full load or no-load conditions in dc grids by disconnectors has been outlined by Vinson [12] previously. Further switching cases such as deparalleling of lines could be considered in the future.

Dielectric properties of the electrode gap after current zero require further investigation, especially for switching cases exciting large transient recovery voltages. Furthermore, influence of the byproducts of disconnector operation onto the solid and gaseous insulation system of the switchgear needs to be minimized as prior research has shown that metallic particles associated with disconnector operation can have detrimental effects on HVdc insulation systems [32], [33].

\section{Conclusions and Outlook}

The presented research demonstrates, that ac disconnectors with bus-transfer switching capability offer an attractive starting point for the design of future disconnectors for use in gas insulated HVdc substations. Bus-transfer of dc currents can be investigated experimentally using the presented test setup as well as by simulating the given equivalent circuit diagram in combination with the previously found extinction criterion. While the magnitude of the bus-transfer current and the length of the bus-transfer loop have been identified as major contributors to the arcing time, the biggest influence onto the reignition behavior at current zero stems from the harmonic content of the bus-transfer current. Therefore, accurate specification of magnitude, frequency and phase angle of current harmonics is vital for the design and testing of disconnectors with dc bustransfer capability.

In future research, applications of disconnectors for switching cases other than bus-transfer in HVdc grids as well as more severe bus-transfer scenarios will be evaluated. Furthermore, methods to reduce arcing times and contact erosion in order to minimize the production of metallic particles are to be investigated.

\section{REFERENCES}

[1] P. Bordignan and G. Bathurst, "Delivery of the Nan'ao multi-terminal VSC-HVDC system," in 11th IET International Conference on AC and DC Power Transmission. Institution of Engineering and Technology, 2015, pp. 1-6.

[2] H. Rao, "Architecture of Nan' ao multi-terminal VSC-HVDC system and its multi-functional control," Power and Energy Systems, CSEE Journal of, vol. 1, no. 1, pp. 9-18, 2015.

[3] C. M. Franck, "HVDC Circuit Breakers: A Review Identifying Future Research Needs," IEEE Transactions on Power Delivery, vol. 26, no. 2, pp. 998-1007, apr 2011.

[4] G. Tang, Z. He, H. Pang, X. Huang, and X.-p. Zhang, "Basic topology and key devices of the five-terminal DC grid," CSEE Journal of Power and Energy Systems, vol. 1, no. 2, pp. 22-35, jun 2015.

[5] G. Tang, "High Power Conversion Technology for High Voltage DC Transmission Application," in IEEE Energy Conversion Congress \& Expo (ECCE) 2016, 2016.

[6] M. Callavik, A. Blomberg, J. Häfner, and B. Jacobson, "Break through! ABB's hybrid HVDC breaker," ABB Review, no. 2, 2013. 
[7] C. Davidson, R. Whitehouse, C. Barker, J.-P. Dupraz, and W. Grieshaber, "A new ultra-fast HVDC Circuit breaker for meshed DC networks," in 11th IET International Conference on AC and DC Power Transmission. Institution of Engineering and Technology, 2015, pp. 047-7.

[8] R. Alvinsson, E. Borg, A. Hjortsberg, T. Hoglund, and S. Hornfeldt, "GIS for HVDC Converter Stations," Cigré Session, no. 14-02-1986, 1986.

[9] M. Mendik, S. Lowder, and F. Elliott, "Long term performance verification of high voltage DC GIS," in 1999 IEEE Transmission and Distribution Conference, vol. 2. IEEE, apr 1999, pp. 484-488 vol.2.

[10] T. Shimato, T. Hashimoto, and M. Sampei, "The Kii Channel HVDC Link in Japan," Cigré Session, no. 14-106-2002, 2002.

[11] Cigré Working Group D1.03, "Gas Insulated Systems for HVDC: DC Stress at DC and AC Systems," Cigré TB506, no. 506, p. 79, 2012

[12] P. Vinson, A. Girodet, A. Hanouna, and S. Poullain, "Investigations of load transfer capabilities of GIS disconnectors for DC grids," in 3rd International Conference on Electric Power Equipment - Switching Technology (ICEPE-ST 2015), 2015.

[13] A. Ritter, M. M. Walter, U. Straumann, and C. M. Franck, "Derivation of Requirements for Small-Current Switching in Future HVDC Substations," in 18th International Symposium on High Voltage Engineering, 2013, pp. 2343-2348.

[14] Cigré Working Group JWG A3/B4.34, "Technical Requirements and Specifications of State-of-the-Art HVDC Switching Equipment," Cigré TB683, Tech. Rep. April, 2017.

[15] A. Ritter, U. Straumann, and C. M. Franck, "Novel Method for Predicting Limit Performance of Bus-Transfer Switching by Disconnectors," IEEE Transactions on Power Delivery, vol. 8977, no. c, pp. 1-1, 2016.

[16] IEC, IEC 62271-102 - Alternating current disconnectors and earthing switches, 1st ed., 2013.

[17] D. F. Peelo, "Current Interruption using High Voltage Air-Break Disconnectors," Ph.D. dissertation, Eindhoven: Technische Universiteit Eindhoven, 2004.

[18] Cigré Working Group A3.28, "Switching Phenomena for EHV and UHV Equipment," Cigré TB570, 2014.

[19] Cigré Working Group JWG 13/14.08, "The current commutation function of HVDC switching devices," Cigré Electra, no. ELT-124-2, 1989

[20] Y. Yokomizu, T. Matsumura, R. Henmi, and Y. Kito, "Total voltage drops in electrode fall regions of SF6, argon and air arcs in current range from 10 to 20000 A," Journal of Physics D: Applied Physics, vol. 29, no. 5, pp. 1260-1267, may 1996.

[21] A. Ritter, L. S. J. Bort, and C. M. Franck, "Five years of pulsed current testing for HVDC switchgear," in 2016 IEEE International Conference on High Voltage Engineering and Application (ICHVE). IEEE, sep 2016, pp. 1-4.

[22] L. Dalessandro, N. Karrer, M. Ciappa, A. Castellazzi, and W. Fichtner, "Online and offline isolated current monitoring of parallel switched highvoltage multi-chip IGBT modules," in 2008 IEEE Power Electronics Specialists Conference. IEEE, jun 2008, pp. 2600-2606.

[23] H. M. Ryan and G. R. Jones, SF6 Switchgear. The Institution of Engineering and Technology, Michael Faraday House, Six Hills Way, Stevenage SG1 2AY, UK: IET, jan 1989, vol. 56, no. 1.

[24] H. Schoetzau, H. Meili, E. Fischer, C. Sturzenegger, and H. Graf, "Dielectric Phase in an SF6 Model Breaker," IEEE Transactions on Power Apparatus and Systems, vol. PAS-104, no. 7, pp. 1897-1902, jul 1985.

[25] J. Mentel, "The Influence of Arc Roots on Current Interruption," in Brown Boveri Symposium on Current Interruption in High-Voltage Networks, K. Ragaller, Ed. Springer, 1978, pp. 119-135.

[26] X. Wang, L. Zhong, M. Rong, A. Yang, D. Liu, Y. Wu, and S. Miao, "Dielectric breakdown properties of hot SF 6 gas contaminated by copper at temperatures of $3003500 \mathrm{~K}$," Journal of Physics D: Applied Physics, vol. 48, no. 15, p. 155205, apr 2015.

[27] S. Yao, R. Huang, X. Zhang, L. Zhong, X. Wang, Y. Wu, and M. Rong, "Influence of copper contamination on dielectric breakdown properties of high-temperature SF6 gas based on Boltzmann equation analysis," in 2015 3rd International Conference on Electric Power Equipment Switching Technology (ICEPE-ST), no. ii. IEEE, oct 2015, pp. 317-320.

[28] H. Saad, Y. Fillion, S. Deschanvres, Y. Vernay, and S. Dennetiere, "On Resonances and Harmonics in HVDC-MMC Station Connected to AC grid," IEEE Transactions on Power Delivery, vol. 8977, no. c, pp. 1-1, 2017.

[29] IEC, IEC/TR 62543 Ed. 1.1 - High-voltage direct current (HVDC) power transmission using voltage sourced converters (VSC), 2013.

[30] Cigré Working Group B4.37, "VSC Transmission," Cigré TB269, 2005.
[31] J. Tepper, M. Seeger, T. Votteler, V. Behrens, and T. Honig, "Investigation on Erosion of $\mathrm{Cu} / \mathrm{W}$ Contacts in High-Voltage Circuit Breakers," IEEE Transactions on Components and Packaging Technologies, vol. 29, no. 3, pp. 658-665, sep 2006.

[32] L. Bing, S. Jian, H. Shangmao, and Z. Ran, "New research progress of HVDC transmission technology in China Southern Power Grid Corporation," in 2016 IEEE International Conference on High Voltage Engineering and Application (ICHVE). IEEE, sep 2016, pp. 1-5.

[33] H. Iwabuchi, S. Matsuoka, A. Kumada, K. Hidaka, Y. Hoshina, T. Yasuoka, and M. Takei, "Influence of tiny metal particles on charge accumulation phenomena of GIS model spacer in high-pressure SF6 gas," IEEE Transactions on Dielectrics and Electrical Insulation, vol. 20, no. 5, pp. 1895-1901, 2013.

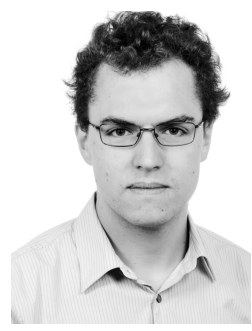

Andreas Ritter (M09) was born in Basel, Switzerland, in 1986. He received the M.Sc. degree in electrical engineering with distinction from ETH Zurich, Zurich, Switzerland, in 2012. He is currently a scientific assistant at the High Voltage Laboratory at ETH Zurich, researching disconnector and earthing switch operation in future multiterminal $\mathrm{HVdc}$ grids.

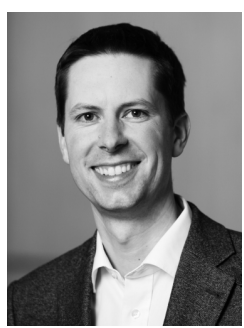

Christian M. Franck (M04 - SM11) received a diploma in physics from the University of Kiel, Germany in 1999 and the Ph.D. degree in physics from the University of Greifswald, Germany in 2003. He was with the Swiss corporate research center of ABB during 2003-2009 as a Scientist and Group Leader for gas circuit breakers and high voltage systems. Currently, he is Associate Professor for High Voltage Technology at the Swiss Federal Institute of Technology (ETH), Zurich, Switzerland. His main research interests are in the area of gaseous insulation systems and equipment for $\mathrm{HVdc}$. 